\title{
Die Krisenreaktion der Vereinten Dienstleistungsgewerkschaft
}

\section{DAS KONZEPT EINER SOZIALEN ANTIKRISENPOLITIK}

Die Vereinte Dienstleistungsgewerkschaft (ver.di) wurde wie andere Gewerkschaften und Akteure vom Ausmaß, der Dynamik und der Tiefe der Weltwirtschaftskrise überrascht. Besonders betroffen waren die Finanzdienstleistungen. Der Zusammenbruch von Lehman Brothers und die faktische Pleite der Hypo Real Estate (HRE) weckten schlimmste Befürchtungen über den drohenden Zusammenbruch des gesamten Bankensystems. Die Geschwindigkeit, mit der auf nationaler, europäischer und internationaler Ebene Rettungspakete für die Banken verabschiedet wurden, beruhigte zwar die Betroffenen. Dennoch war für ver.di klar, dass diese Krise ein grundlegendes Umdenken und Umsteuern verlangte. „Die Notwendigkeit des Rettungspaketes verdeutlicht auf dramatische Weise: Die neoliberale Politik der Deregulierung und Liberalisierung ist grundlegend gescheitert: Jetzt ist die deutliche Umkehr auf allen Politikfeldern notwendig. " ${ }^{1}$

Nachdem im Oktober 2008 die weltweite Nachfrage nach Automobilen und anderen Industriegütern dramatisch einbrach, verlagerte sich die öffentliche Aufmerksamkeit auf diese Leitbranchen der deutschen Volkswirtschaft. Betroffen waren die industrielle Produktion, aber auch die industrienahen Dienstleistungen in der Transport- und Logistikbranche und vor allem die Arbeitnehmerüberlassung. Die Zahl der Leiharbeiter verringerte sich um rund 300.000 in wenigen Monaten.

Für die ver.di bestätigten sich die Einschätzungen jener Ökonomen, die bereits vor Ausbruch der Wirtschaftskrise auf die drohenden Risiken weltweiter Ungleichgewichte für die deutsche Volkswirtschaft hingewiesen und vor allem auf eine Ankurbelung der Binnenmarktnachfrage und die Steigerung der öffentlichen Investitionen gedrängt hatten. Ver.di kritisierte daher auch den mehr als zögerlichen Vorstoß der Bundesregierung, ein erstes Konjunkturpaket im Umfang von 4 Mrd. € aufzulegen („ein Pups“so Frank Bsirske im Rundfunk).
Umso dringlicher wurde aber ein eigenes Antikrisenkonzept, das sowohl der dramatischen Entwicklung auf den Weltfinanzmärkten wie dem rapiden Wachstumseinbruch gerecht würde.

Am 5. Dezember 2008 verabschiedete der Gewerkschaftsrat - das höchste Gremium zwischen den ver.di-Kongressen - die Resolution „Aktiv werden für eine soziale Antikrisenpolitik“.2 Entsprechend den wirtschaftspolitischen Linien, die ver.di vertritt, wurde in dieser Resolution die Krise einerseits als Konjunkturkrise, wie „sie seit dem 19. Jahrhundert die kapitalistische Wirtschaftsentwicklung prägen“ (ebd.), beschrieben. Gleichwohl aber wurde auch ihre spezifische Dimension als „weltweite Krise der Finanzmärkte und Banken“ (ebd.) erfasst und konstatiert: „Der letzte Grund der Finanzkrise ist allerdings der gewaltige Überschuss an Anlage suchendem Kapital, der zu gigantischen Spekulationsblasen führt, die irgendwann platzen müssen“ (ebd.).

Gefordert wurden die umfassende Reregulierung der Finanzmärkte, Transparenz und Aufsicht sowie groß dimensionierte Konjunkturprogramme, um so „die realwirtschaftliche Nachfrage zu stabilisieren und die Arbeitsplätze zu schützen“ (ebd.).

Diese beiden wesentlichen strategischen Achsen der Regulierung der Finanzmärkte und der antizyklischen Konjunkturpolitik wurden ergänzt um die Forderung nach einer Sozialstaatsoffensive auf nationaler und europäischer Ebene mit dem Ziel, „die öffentliche Daseinsvorsorge und Daseinsfürsorge in öffentlicher Verantwortung und in öffentlichem Eigentum wieder auszubauen und professionell $\mathrm{zu}$ organisieren“ (ebd.).

Forderungen nach einer neuen solidarischen Weltwirtschaftsordnung, die Hunger überwindet, sowie nach weltweiten nachhaltigen Anstrengungen, um die „Energieversorgung, die Produktions- und Lebensweise ökologisch verträglich umzubauen, “ mündeten in dem Vorschlag nach einem neuen, sozial-ökologischen Green New Deal. Die „Beendigung der
Vorherrschaft des Finanzkapitals über die Wirtschaft" und „die Erweiterung der demokratischen Gestaltung der Verhältnisse durch die Menschen selbst" gaben dem Antikrisenprogramm eine über die aktuelle Krisenbewältigung hinausgreifende systemüberwindende Dimension.

Die soziale Dimension des Antikrisenprogramms spiegelt sich wider in den Forderungen:

- Ausgabe von Barschecks und Gutscheinen von $500 €$ mit sozial gestaffelten Bedingungen,

- Reform der Lohn- und Einkommensteuer mit einer Entlastung der Durchschnittsverdiener von $1.000 €$ pro Jahr,

- dauerhafte Besteuerung großer Vermögen und Erbschaften und Anhebung des Spitzensteuersatzes,

- Anhebung des Arbeitslosengeldes (ALG) II auf $420 €$,

- gesetzlicher Mindestlohn von 7,50 bis $9 €$,

- Einlösung des Grundsatzes „Gleicher Lohn für gleiche Arbeit in der Leiharbeit“, - Rücknahme der Rente mit 67,

- Verkürzung der faktischen Höchstarbeitszeit auf 40 Stunden europaweit sowie weitere öffentlich geförderte Arbeitszeitverkürzungen.

\section{GESPALTENE KRISENWAHRNEHMUNC}

Am 12. Dezember 2008 fand im Bundeskanzleramt die entscheidende Gesprächsrunde statt, in der die Weichen für die

\footnotetext{
Gemeinsamer Brief vom 5. November 2008 des ver.di-Vorsitzenden Frank Bsirske und von Uwe Foullong, ver.di Vorstand, an die ver.di-Mitglieder in den Finanzdienstleistungen.

2 "Aktiv werden für eine soziale Antikrisenpolitik" Resolution des ver.di-Gewerkschaftsrates zur Wirtschafts- und Finanzkrise, www.wipo.verdi. de/Dokumente.
}

Wolfgang Uellenberg-van Dawen, $D r$. ist Leiter des Ressorts 1 "Politik und Planung" in der ver.di Bundesverwaltung. e-mail:wolfgang.uellenberg@verdi.de 
Antikrisenpolitik der Bundesregierung gestellt wurden. Dem öffentlichen Druck, der sogar aus dem Arbeitgeberlager kam, sowie den Forderungen aus den Koalitionsparteien und Gewerkschaften nachgebend, gab die große Koalition ihren Widerstand gegen eine antizyklische Konjunkturpolitik (Steinbrück: „Strohfeuer“) auf und brachte ein Konjunkturpaket im Gesamtvolumen von 80 Mrd. $€$ auf den Weg. Dieses Paket umfasste einerseits ein öffentliches Investitionsprogramm von rund $22 \mathrm{Mrd}$. $€$ (inkl. der Maßnahmen des ersten Konjunkturpaketes) sowie umfangreiche Senkungen der Einkommensteuern und einen Kindergeldzuschlag von $100 €$ für alle. Ver.di begrüßte das Investitionsprogramm, von dem vor allem die Kommunen und Länder in den Bereichen Bildung und Umwelt profitieren würden, warnte aber vor der Schwächung der Finanzkraft der öffentlichen Haushalte durch weitere lineare Steuersenkungen. ${ }^{3}$

Als im 1. Quartal 2009 alle gesetzgeberischen Entscheidungen gefallen waren und das Konjunkturpaket II umgesetzt werden konnte, fiel die Wachstumsrate auf unter $5 \%$. Der Auftragseinbruch in vielen Industriebranchen war dramatisch. Nur die Abwrackprämie federte die Gefahr eines kompletten Produktionsstillstands in der Automobilindustrie ab. Mit dem Abbau von Arbeitszeitkonten und der erweiterten Kurzarbeit wurden Massenentlassungen verhindert.

Demgegenüber waren tiefe Einbrüche im Konsumverhalten über die Jahreswende ausgeblieben, da die relativ günstigen Tarifabschlüsse der ersten Jahreshälfte 2008 ihre Wirkung entfalteten. Auch die Gebietskörperschaften verzeichneten noch keine Einnahmeausfälle, da die Gewerbesteuereinnahmen noch aus 2008 hoch waren.

Vor diesem Hintergrund empfanden noch im Jahr 2009 Umfragen zufolge auBerhalb der von der Krise der Industrie direkt betroffenen Regionen rund Zweidrittel der Bevölkerung die Krise nicht als bedrohlich.

Wahrscheinlich war dies eine Ursache für die - angesichts der Erwartungen der Initiatoren - geringe Mobilisierung zu den Demonstrationen, die im März 2009 in Berlin und Stuttgart stattfanden unter dem Motto: „Wir zahlen nicht für eure Krise“. Im Deutschen Gewerkschaftsbund (DGB) hatten die Gewerkschaften darauf gedrungen, eine klare Positionierung vorzuneh- men. Zum DGB-Kapitalismuskongress am 14. und 15. Mai 2009 legte ver.di mit ihren Thesen eine umfassende Analyse der Ursachen und der Dimensionen der Krise vor. ${ }^{4}$ Zur Demonstration am 16. Mai 2009 in Berlin hatte auch ver.di bundesweit intensiv mobilisiert, aber eine gesellschaftliche Protestbewegung gegen die Verursacher der Krise kam nicht zustande.

Die Erwartungen der ver.di wie aller Gewerkschaften, dass nun auch die Politik in der Krise ihre wirtschafts- und finanzpolitische Strategie umfassender und grundlegender ändern würde, wurden enttäuscht: Weder fielen auf den G-20Gipfeln die notwendigen Beschlüsse, um zu einer schnellen und durchgreifenden Regulierung der Finanzmärkte zu kommen, noch fanden sich die europäischen Institutionen und Staaten zu einer gemeinsamen abgestimmten Antikrisenpolitik auf europäischer Ebene zusammen. Die Bundesregierung traf mit der Einführung der Schuldenbremse ins Grundgesetz eine Entscheidung von weitgehender Tragweite, da diese eine schuldenfinanzierte Politik gegen die Krise abrupt abbrechen würde, bevor sich Wachstumseffekte einstellen würden. Die Kritik der ver.di, des DGB, aber auch namhafter Ökonomen wie des Wirtschaftsweisen Peter Bofinger sowie zahlreicher Bundes- und Landespolitiker konnten den Bundesfinanzminister nicht umstimmen: Seine Angst vor einer inflationären Entwicklung sowie der Druck der nach wie vor monetaristisch ausgerichteten Finanzmärkte und ihrer medialen Unterstützer wischten alle Einwände gegen die Schuldenbremse vom Tisch. ${ }^{5}$

\section{FORDERUNG NACH EINEM DRITTEN KONJUNKTURPAKET}

Während die Bundesregierung die Wende nach rückwärts, hin zu den Dogmen der monetaristischen Politik, vollzog, brach das Wachstum weiter ein: Vor diesem Hintergrund legte ver.di das Konzept für ein Konjunkturpaket III vor. Dieses Konjunkturpaket III sollte nicht mehr allein durch eine antizyklische Konjunkturpolitik Wachstum generieren und damit etwa 2 Mio. Arbeitsplätze schaffen, sondern darüber hinaus die Weichen für einen sozialen und ökologischen Umbau der Wirtschaft stellen: ${ }^{6}$

Angesichts eines Exporteinbruchs von $20 \%$ soll durch eine Ausweitung der öffentlichen Investitionen auf $75 \mathrm{Mrd}$. $€$ jähr- lich sowie eine Steigerung der Konsumkraft vor allem der Arbeitslosen und Geringverdiener im Umfang von $25 \mathrm{Mrd}$. $€$ die Konjunktur angekurbelt werden.

Mit den Investitionsfeldern sollte zugleich ein nachhaltiges und sozialökologisch ausgerichtetes Wachstum ermöglicht werden: $30 \mathrm{Mrd}$ € jährlich in Kindertagesstätten, Ganztagsschulen und Hochschulen, $20 \mathrm{Mrd}$. $€$ für Ver- und Entsorgung und energetische Gebäudesanierung; $5 \mathrm{Mrd}$. $€$ für die ökologische Modernisierung des Verkehrs, insbesondere des Öffentlichen Personennahverkehrs (ÖPNV), der von der Bundesregierung völlig aus dem Förderprogramm des Konjunkturpaketes II ausgeklammert worden war; 15 Mrd. $€$ zur Beseitigung eines riesigen Investitionsstaus in den Krankenhäusern und bei der Altenpflege sowie $5 \mathrm{Mrd}$. $€$ für den Ausbau des Breitbandnetzes. Investitionsmittel sollten aber nicht nur für „Beton“, sondern auch für das dringend benötigte Personal ausgegeben werden.

Das arbeitsmarktpolitische Sofortprogramm konzentrierte sich auf die generelle Verlängerung des ALG-I-Bezugs auf ein Jahr, unabhängig von den unterschiedlichen Anwartschaften, und auf zwei Jahre für alle über 50-Jährigen, die Erleichterung der Kurzarbeit, die Erhöhung des ALG II und der Grundsicherung auf $435 €$ sowie die Verlängerung der Altersteilzeit, um Auszubildende zu übernehmen. Finanziert werden sollte das Gesamtpaket durch eine sozial gerechte Steuerpolitik ${ }^{7}$, für die der Gewerkschaftsrat bereits votiert hatte. $\mathrm{Zu}$ dem nahm der ver.di-Bundesvorstand die Forderung der IG Metall nach einem Public Equity-Fonds in Höhe von $100 \mathrm{Mrd}$. zur Rettung bedrohter Industrieunternehmen in sein Forderungspaket auf.

3 Vgl. ver.di (2009): Konjunkturpaket II. Mehr Klientelpolitik als ein Programm zur Krisenbekämpfung, in: Wirtschaftspolitische Informationen 1, www.wipo.verdi.de.

4 Ver.di Bundesvorstand, Bereich Politik und Planung (2009):Thesen zum DGB Kapitalismuskongress, in: perspektiven.verdi.de.

5 Ver.di Bundesvorstand (2009): Investitionen in die Zukunft statt Schuldenbremse, Mai.

6 Ver.di Bundesvorstand (2009): Solidarisch aus der Krise, Wirtschaftspolitik, Mai.

7 Dazu zählen: Eine Vermögenssteuer von $1 \%=$ $20 \mathrm{Mrd}$. $€$, eine höhere Erbschaftssteuer $=6 \mathrm{Mrd}$. $€$, eine höhere Unternehmenssteuer $=20 \mathrm{Mrd}$. $€$, eine Gemeindewirtschaftssteuer $=3 \mathrm{Mrd}$. $€$, die Finanztransaktionssteuer von $10 \mathrm{Mrd}$. $€$ sowie ein besserer Steuervollzug und mehr Einnahmen aus der Einkommensteuer von insgesamt $16 \mathrm{Mrd}$ €. 


\section{MINDESTLOHN UND GUTE ARBEIT}

Mit der Forderung nach einem dritten Konjunkturpaket befand sich ver.di schon mitten im Bundestagswahlkampf. Während die FDP in ihrem Wahlprogramm dem neoliberalen Fundamentalismus mit sozialem Anstrich huldigte, verhielt sich die Union weitgehend uneindeutig. Einzig in der Ablehnung eines allgemeinen gesetzlichen Mindestlohnes positionierte sie sich unmissverständlich. In den Wahlprogrammen von SPD, Bündnis 90/Die Grünen sowie der Partei Die Linke wurden die ver.di-Konzepte in unterschiedlichem Maße aufgenommen. Einigendes Band in allen Parteiprogrammen von RotRot-Grün war die Forderung nach einem allgemeinen gesetzlichen Mindestlohn zwischen 7,50 $€$ (SPD und Grüne) bis zu $10 €$ (Die Linke). Die Wahlkampagne der ver.di (gemeinsam mit der Gewerkschaft Nahrung, Genuss, Gaststätten (NGG) und unterstützt vom DGB) „Deine Stimmen für den Mindestlohn“ konzentrierte sich auf diese Forderung.

Tarifpolitisch hatte ver.di im Jahre 2009 ihr Ziel, die Massenkaufkraft zu stärken, erreichen können: Nach Warnstreiks bei der Telekom, der Druckindustrie, im Einzelhandel sowie im öffentlichen Dienst der Länder konnten Einkommensverbesserungen zwischen 2 und $5 \%$ (TD Länder wie TVöD Bund-Kommunen) durchgesetzt werden. Banken und Versicherungen vereinbarten Lohnerhöhungen von $2 \%$. Große Beachtung fand der wochenlange Arbeitskampf im Sozial- und Erziehungsdienst. In dieser Auseinandersetzung wollte ver.di die Anerkennung und Aufwertung der Tätigkeit der Beschäftigten in Kindertagesstätten und Jugendämtern durchsetzen, die im Rahmen der Anpassung der Entgelte beim Übergang vom BAT zum TVöD über Jahre von den Arbeitgebern beharrlich verweigert wurden. Dieser Arbeitskampf machte aber nicht eine materielle Geldforderung zum Gegenstand der Auseinandersetzung. Vielmehr ging es um den Stellenwert der Erziehungs- und Bildungsarbeit sowie der Jugendsozialarbeit und der allgemeinen Sozialarbeit für die Gesellschaft wie auch um die gesundheitlichen Auswirkungen schlechter Arbeitsbedingungen auf die Betroffenen. Trotz mancher verärgerter Eltern fanden die Tarifbewegung und der Streik eine breite öffentliche Unterstützung - bis hin zu Spitzenpolitikern von Koalition und Opposition.

Diese Tarifbewegung stellte wie auch die folgende z. B. im Bereich der Banken das Thema "Gute Arbeit" stärker in den Mittelpunkt. Obwohl die Arbeitgeberverbände wie auch die politischen Parteien mit Ausnahme der FDP von einer Deregulierung der Arbeitsverhältnisse und der Schwächung der Mitbestimmung Abstand nahmen, wuchs und wächst der Druck von Arbeitgebern auf die Beschäftigten, Lohneinbußen und Arbeitsverdichtungen wie Arbeitszeitverlängerungen hinzunehmen. Unter dem Motto „Hauptsache Arbeit“ werden so viele Beschäftigte mit den Kosten der Krise belastet. Dem stellen ver.di und die anderen DGB Gewerkschaften das Konzept der Guten Arbeit entgegen. Gute Arbeit als Anspruch der Beschäftigten auf sichere, gut entgoltene, sinnvolle, qualifizierte, anerkannte und mitbestimmte Arbeit soll über Aktivitäten in den Betrieben und Verwaltungen und in den Tarifbewegungen zum einen dazu beitragen, die Beschäftigten vor einer zusätzlichen krisenbedingten Ausbeutung ihrer Arbeitskraft zu schützen. Zum anderen aber zielt die Kampagne Gute Arbeit darauf ab, als Bestandteil einer sozialen und ökologischen Strategie gegen die Krise die Auseinandersetzung um die Qualität der Arbeit aufzunehmen.

\section{ZUSPITZUNG DER VERTEILUNGSFRAGE}

Mit dem Ausgang der Bundestagswahl und anschließend sichtbar gewordenen Politikmustern der neuen CDU/FDP-Koalition haben die von ver.di entwickelten Antikrisenkonzepte eine neue Zuspitzung erfahren. Denn das Programm der Koalition zielt nach einer Übergangsphase, die der Tiefe der Krise geschuldet ist, auf eine Rückkehr zum neoliberalen Dogma: Auf der einen Seite Steuersenkungen vor allem für einkommensstarke Schichten und - wie mit der geplanten Abschaffung der Gewerbesteuer beabsichtigt - für Unternehmen, auf der anderen Seite radikaler Schuldenabbau zulasten der Langzeitarbeitslosen und der Sozialen Sicherungssysteme. Trotz der in der Krise gewachsenen und öffentlich verbreiteten Erkenntnis, dass eine andere
Verteilungspolitik notwendig ist, will diese Koalition, wenn auch mit unterschiedlicher Geschwindigkeit der Koalitionspartner, zurück zu einer traditionellen Umverteilungspolitik von unten nach oben. ${ }^{8}$

Damit wurde und wird die Verteilungsfrage wieder in den Mittelpunkt der öffentlichen Auseinandersetzung gestellt. Diese Auseinandersetzung spitzt sich fast täglich mehr zu durch den massiven Einbruch der öffentlichen Einnahmen. Jetzt zeigen die Einkommensteuersenkungen des Konjunkturpaketes II ebenso ihre negativen Wirkungen wie der Einbruch der Gewerbesteuereinnahmen infolge der Wirtschaftskrise. Mit dem sogenannten Wachstumsbeschleunigungsgesetz hat die Koalition sich zudem die öffentliche Blöße gegeben, zugunsten ihrer Klientel die öffentlichen Kassen in Zeiten der Krise weiter zu plündern.

Während Anzeichen einer Erholung der Realwirtschaft erkennbar sind, hat auf den internationalen Finanzmärkten eine neue und bisher nicht gekannte Spekulationswelle gegen die Europäische Währung begonnen, der die Staaten der Euro-Zone trotz eines kurzfristigen Interventionsprogramms von $750 \mathrm{Mrd}$. $€$ bei Weitem noch nicht Herr geworden sind. Weil die Finanzmärkte weitgehend unreguliert blieben, sich wirtschaftliche Ungleichgewichte in Europa weiter verschärft haben, steht nun vor allem Europa eine zweite und tiefe Welle der Krise bevor.

Waren noch vor einem Jahr Zweidrittel der Menschen in Deutschland der Meinung, die Krise berühre sie nicht, so hat sich dieses Verhältnis umgekehrt. Nun sind Zweidrittel der Meinung, dass sie von der Krise direkt und indirekt betroffen sind. Die Angst vor Arbeitslosigkeit wächst, umgekehrt sinkt das Vertrauen in die Politik insgesamt. Noch ist aus der Wirtschaftskrise keine Krise des Sozialstaates und der Demokratie geworden, aber es fehlt nicht mehr viel dazu. Für die Gewerkschaften kommt es nun nicht mehr so sehr darauf an, abermals Konzepte zu entwickeln, sondern für die Durchsetzung ihrer vorhandenen, aber nicht eingelösten Forderungen Mehrheiten in den Betrieben und Verwaltungen wie auch Mehrheiten in der Gesellschaft zu finden und sie zu mobilisieren.

8 Vgl. hierzu: ver.di (2009): Bewertung des Koalitionsvertrages, in: Politik von A-Z, www.verdi.de. 